\title{
On the formation of crack networks in high cycle fatigue
}

\section{De la formation de réseaux de fissures en fatigue à grand nombre de cycles}

\author{
Nicolas Malésys ${ }^{\mathrm{a}, \mathrm{c}}$ Mohammad Seyedi ${ }^{\mathrm{a}, \mathrm{b}}$ Ludovic Vincent ${ }^{\mathrm{c}}$ François Hild $^{\mathrm{a}}$ \\ ${ }^{a} L M T$-Cachan, ENS de Cachan / CNRS UMR 8535 / Université Paris 6 \\ 61 Avenue du Président Wilson, F-94235 Cachan Cedex, France \\ ${ }^{\mathrm{b}}$ EDF-R\&D, Département AMA, 1 Avenue du Général de Gaulle, F-92141 Clamart Cedex, France. Now at \\ BRGM/ARN, 3 Avenue Guillemin, F-45060 Orléans Cedex, France \\ ${ }^{\mathrm{c}}$ CEA Saclay, DEN-DANS/DMN/SRMA/LC2M, F-91191 Gif sur Yvette Cedex, France
}

\begin{abstract}
A probabilistic model based on an initial distribution of sites is proposed to describe different aspects of the formation, propagation and coalescence of crack networks in thermomechanical fatigue. The interaction between cracks is modeled by considering shielding effects.
\end{abstract}

\section{Résumé}

La création de fissures, leur propagation et coalescence ultérieure dans un réseau sont traitées à l'aide d'un modèle probabiliste. Les interactions entre fissures sont prises en compte par la description des effets d'écran.

Key words: Fatigue; crack shielding; Poisson point process; Weibull model

Mots-clés : Fatigue; effet d'écran; processus ponctuel de Poisson; modèle de Weibull

Email addresses: malesys@lmt.ens-cachan.fr (Nicolas Malésys), m.seyedi@brgm.fr (Mohammad Seyedi), ludovic.vincent@cea.fr (Ludovic Vincent), hild@lmt.ens-cachan.fr (François Hild). 


\section{Version française abrégée}

Le modèle proposé dans cette Note traite de manière unifiée de trois échelles de fissuration en fatigue [2]. L'échelle microscopique est liée au stade I de propagation. L'échelle mésoscopique correspond à la propagation en stade II des fissures. Enfin, la coalescence des fissures se produit à l'échelle macroscopique. Le stade II sera le plus important dans cette étude. La microstructure est modélisée par des sites sur lesquels des fissures peuvent s'amorcer par l'intermédiaire d'un processus ponctuel de Poisson.

En fatigue à grand nombre de cycles, de toutes les microfissures, seule une fraction peut former des mésofissures. Un processus de germination continue est introduit. Une microfissure peut conduire à l'amorçage d'une mésofissure à condition qu'elle ne soit pas écrantée par une autre mésofissure. Un domaine d'obscurcissement autour de mésofissures (i.e., une zone dans laquelle les contraintes sont inférieures au niveau appliqué, ce qui empêche tout nouvel amorçage) est défini. Ainsi, de nouvelles mésofissures sont amorcées s'il existe un site potentiel dans la zone considérée et, lorsque la condition d'amorçage est satisfaite, et si cette fissure n'est pas écrantée par les mésofissures existantes [figure 1(a)]. L'élément fondamental pour analyser l'amorçage est l'horizon [figure 1(b)]. Il s'agit de regarder dans le passé de l'histoire de chargement. Une microfissure en $\mathrm{S}$ peut amorcer une mésofissure si aucune mésofissure ne se trouve dans son horizon. L'incrément de densité de mésofissures $\lambda_{m}$ est calculé à l'aide de la probabilité d'obscurcissement $P_{\text {obs. }}$. À partir de cette information, un modèle d'endommagement est écrit à l'échelle mésoscopique. Enfin, la coalescence est alors être décrite à l'aide d'une condition de localisation de l'endommagement.

Various components in nuclear power plants are subjected to thermomechanical loadings during their lifetime. Thermal striping was observed in the mixing zones of the auxiliary cooling system of nuclear power plants [1]. This is not troublesome as long as a fracture analysis indicates that the cracks will not grow notably during the remaining service life. Consequently, the evaluation of crack initiation, their subsequent propagation and coalescence in structures subjected to thermomechanical loadings is very important to determine investigation periods and maintenance programs. It is proposed to analyze the three stages of cracking by using a unified probabilistic framework.

\section{Initiation, propagation and coalescence in a crack network}

The following model aims at bridging three scales [2]. First the microscale, which is related to stage I of the fatigue process, depends upon the details of the microstructure. The cracks are considered as microstructurally short (they will be referred to as microcracks). This scale ends when mesocracks are initiated. The second scale corresponds to the propagation of mesocracks (i.e., stage II fatigue) that form the network. The cracks are considered as physically small. Last, the third scale is concerned with coalesced mesocracks that form a macrocrack (i.e., a long crack). Since stage II fatigue is predominant, the cracking directions are assumed to be aligned along the principal stress directions $\mathbf{d}_{i}$ (here constant during the whole load history). Each direction will be considered independently. The stress $\sigma$ will denote any of the in-plane local principal stresses $\sigma_{1}, \sigma_{2}$ since initiation is assumed to occur on the surface of samples or structures.

The microstructure is modeled in terms of sites where cracks may initiate. The sites are approximated by points of density $\lambda_{t}$ (i.e., their average number per unit surface or length). For example, these points have a random yield stress $\sigma_{y}$ accounting for microplasticity in their vicinity. In the present setting, they correspond to the distribution of local fatigue limits $\Delta \sigma_{\infty}\left(=\sigma_{y}\right)$. A Poisson point process of intensity $\lambda_{t}$ is considered herein. A power law function is assumed and leads to a Poisson-Weibull model [3] 


$$
\lambda_{t}(\Delta \sigma)=\lambda_{0}\left(\frac{\Delta \sigma}{\Delta \sigma_{0}}\right)^{m},
$$

where $m$ is the Weibull modulus (i.e., it characterizes the scatter in local fatigue limits or similarly in yield stress level), $\Delta \sigma$ the stress amplitude, and $\Delta \sigma_{0}$ the scale parameter relative to a reference density $\lambda_{0}$. The probability $P$ of finding $N_{\mu}=\nu$ microcracks within a uniformly loaded domain $\Omega$ is expressed in terms of a Poisson distribution

$$
P\left(N_{\mu}=\nu, \Omega\right)=\frac{\left[\lambda_{t}(\Delta \sigma) Z\right]^{\nu}}{\nu !} \exp \left[-\lambda_{t}(\Delta \sigma) Z\right] .
$$

The product $\lambda_{t}(\Delta \sigma) Z$ corresponds to the average number of microcracks in a domain $\Omega$ of measure $Z$. With a weakest link hypothesis, a two-parameter Weibull law [4] is retrieved [i.e., the failure probability $P_{F}$ is given by $\left.P_{F}=1-P\left(N_{\mu}=0, \Omega\right)=1-\exp \left\{-\lambda_{t}(\Delta \sigma) Z\right\}\right]$. The Weibull parameters may therefore be determined by analyzing endurance data for which the majority of the number of cycles is used in the formation of a mesocrack.

In high cycle fatigue, among all these microcracks, there is only a fraction for which the mesoscopic initiation condition is satisfied. Let $\lambda_{t I}$ denote the corresponding density that depends upon the stress amplitude $\Delta \sigma$ and the number of cycles $N$. For instance, a threshold $\Delta \sigma_{u}(N)$ accounting for continuous mesoscopic initiation is considered

$$
\lambda_{t I}(N ; \Delta \sigma)=\lambda_{t}\left[\left\langle\Delta \sigma-\Delta \sigma_{u}(N)\right\rangle\right] .
$$

where $\langle\bullet\rangle$ are the Macauley brackets. Equation (3) shows that the initiation process needs a minimum number of cycles $N_{\min }$ (i.e., such that $\left.\Delta \sigma-\Delta \sigma_{u}\left(N_{\min }\right)=0\right)$ to initiate the first mesocrack. In Eqn. (3), the principal variable is $N$ and $\Delta \sigma$ appears as a parameter. Similarly, with a weakest link hypothesis, a three-parameter Weibull law is retrieved as long as $\Delta \sigma_{u}>0$. To be consistent with the previous analysis, it is assumed that $\Delta \sigma_{u}(N \rightarrow+\infty) \rightarrow 0$ so that high cycle fatigue (i.e., $N<+\infty$ ) and endurance (i.e., $N \rightarrow+\infty)$ are described in the same framework. Therefore, $\Delta \sigma_{\infty}+\Delta \sigma_{u}(N)$ corresponds to the equation of a constant failure probability in a Woehler diagram.

To understand why a microcrack may initiate a mesocrack, one has to model its interaction with other mesocracks. An obscuration domain of measure $Z_{o b s}$ around mesocracks (i.e., a zone in which the stresses are less than the applied stresses, thus do not allow for new initiations) has to be defined. The obscuration domain of measure $Z_{\mathrm{obs}}$ is assumed to be proportional to the current size $a$ of propagating mesocracks

$$
Z_{\text {obs }}\left(N-N_{I} ; \Delta \sigma\right)=S\left[a\left(N-N_{I} ; \Delta \sigma\right)\right]^{n},
$$

where $N_{I}$ the number of cycles to mesoscopic initiation, $n=1$ or 2 the space dimension, $S$ a shape parameter independent of the Weibull modulus $m$ but dependent on the space dimension $n$ [5]. It may be noted that the initial mesocrack size $a(0 ; \Delta \sigma)$ is different from zero and depends upon microstructural parameters [2]. By using this set of hypotheses, microcracks do not obscure each other and mesocracks obscure microcracks, thereby partly inhibiting mesocrack initiation, and some mesocracks.

New mesocracks will be initiated only if a microcrack exists in the considered zone, if the initiation condition is met and if the crack does not belong to any relaxed zone depicted in gray in Fig. 1(a). The spatial position of the microcracks is represented as a simple abscissa (instead of a two- or one-dimensional representation) of an $x-y$ graph where the $y$-axis denotes the number of cycles. The microscopic growth is depicted by the black zones. A first mesocrack initiation occurs at point 1 for a number of cycles equal to $N_{1}$. The initiated mesocrack creates a stress relaxation zone or an "obscured zone." For a number of cycles $N_{3}$, the second mesocrack will be initiated at point 3, which is outside the obscuration zone of mesocrack 1. The second initiated mesocrack creates its own obscured zone. The sites 2 and 4 do not create mesocracks because they are obscured by the first and second mesocracks. The space-time (i.e., 


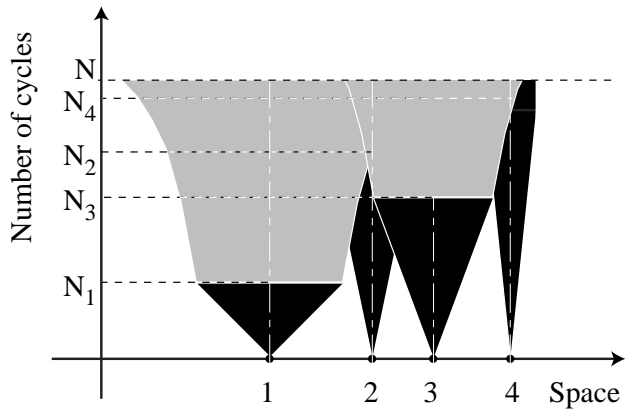

$-\mathrm{a}-$

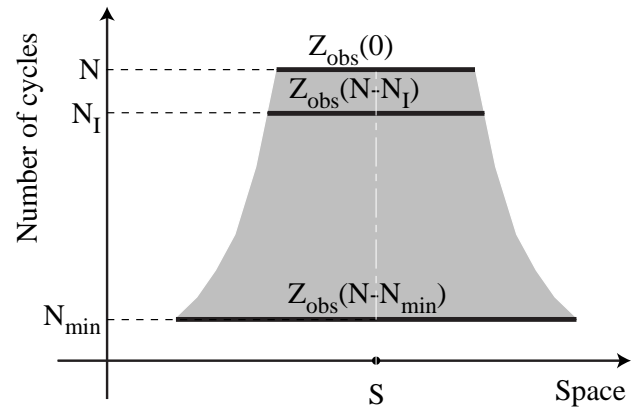

$-b-$

Figure 1. -a-Depiction of two mesocrack propagations (1 and 3) and obscuration for two microcracks (2 and 4). -b-Horizon for a given location S. -a-Schématisation de la propagation de deux mésofissures (1 et 3 ) et de l'écrantage de deux microfissures (2 et 4). -b-Horizon en un lieu donné S.

number of cycles) diagram is composed of the union of the obscured zones where no crack initiate and their complementary zones where any active site initiates a mesocrack. The key element to analyze the interaction between existing mesocracks and microcracks is the horizon of a given site $\mathrm{S}$ [Fig. 1(b)]. It consists in looking at the past of S. A microcrack S will initiate a mesocrack if it is not obscured by other mesocracks. The horizon is a space-time zone where $\mathrm{S}$ is at least obscured by another mesocrack. Consequently, for a mesocrack to be formed, its horizon should not contain any mesocrack. In the present setting, obscuration occurs at the mesoscopic level. Therefore, the density $\lambda_{t I}$ is split into two parts, namely, $\lambda_{m}$, the density of mesocracks and the obscured density. The increment of $\lambda_{m}$ is related to that of $\lambda_{t I}$ by

$$
\frac{\mathrm{d} \lambda_{m}}{\mathrm{~d} N}(N ; \Delta \sigma)=\frac{\mathrm{d} \lambda_{t I}}{\mathrm{~d} N}(N ; \Delta \sigma) \times\left[1-P_{\mathrm{obs}}(N ; \Delta \sigma)\right]
$$

with $\lambda_{m}(0 ; \Delta \sigma)=0$ and $P_{\text {obs }}$ the probability of obscuration

$$
P_{\mathrm{obs}}(N ; \Delta \sigma)=1-\exp \left[-\widehat{Z}_{\mathrm{obs}}(N ; \Delta \sigma) \lambda_{t I}(N ; \Delta \sigma)\right],
$$

where $\widehat{Z}_{\mathrm{obs}}$ is the measure of the mean obscuration zone [6]

$$
\widehat{Z}_{\mathrm{obs}}(N ; \Delta \sigma) \lambda_{t I}(N ; \Delta \sigma)=\int_{N_{\min }}^{N} Z_{\mathrm{obs}}\left(N-N_{I} ; \Delta \sigma\right) \frac{\mathrm{d} \lambda_{t I}}{\mathrm{~d} N_{I}}\left(N_{I} ; \Delta \sigma\right) \mathrm{d} N_{I}
$$

It is worth noting that Eqn. (6) accounts for overlappings of obscuration zones. Furthermore, in the context of mathematical morphology, the above-described approach corresponds to a Boolean islands model [7]. By using Eqn. (3), Eqn. (7) is rewritten in terms of the underlying distribution of endurance limits $\Delta \sigma_{\infty}$

$$
\widehat{Z}_{\mathrm{obs}}(N ; \Delta \sigma) \lambda_{t}\left[\left\langle\Delta \sigma-\Delta \sigma_{u}(N)\right\rangle\right]=\int_{0}^{\left\langle\Delta \sigma-\Delta \sigma_{u}(N)\right\rangle} Z_{\mathrm{obs}}\left[N-N_{I}\left(\Delta \sigma_{\infty}\right) ; \Delta \sigma\right] \frac{\mathrm{d} \lambda_{t}}{\mathrm{~d} \Delta \sigma_{\infty}}\left(\Delta \sigma_{\infty}\right) \mathrm{d} \Delta \sigma_{\infty},
$$

where $N_{I}\left(\Delta \sigma_{\infty}\right)$ corresponds to the number of cycles to initiation associated with $\Delta \sigma_{\infty}\left[\right.$ e.g., $N_{I}(0)=$ $N_{\text {min }}$ ]. When the crack propagation law is known, the change of $Z_{\text {obs }}$ is written [see Eqn. (4)] and the current density of mesocracks is derived. 
From this information, a damage model at the mesoscale is written. To each principal direction $\mathbf{d}_{i}$ is associated a damage variable $D_{i} \equiv P_{\mathrm{obs}}\left(N ; \Delta \sigma_{i}\right)$ that characterizes the overall mesocracking state. The "effective" stress $\Delta \sigma_{i}$ is here taken equal to $\sqrt{2 E \Delta Y_{i}}$, where $\Delta Y_{i}$ is the amplitude of the thermodynamic force associated with the damage variable $D_{i}, E$ the Young's modulus of the virgin material. The mesoscopic principal stress amplitudes $\Delta \Sigma_{i}$ are related to the corresponding strain amplitudes $\Delta \epsilon_{i}$ by the reduced stiffness tensor $\mathcal{K}$ that depends upon the two damage variables. Its inverse reads

$$
\mathcal{K}^{-1}\left(D_{1}, D_{2}\right)=\frac{1}{E}\left[\begin{array}{cc}
\frac{1}{1-D_{1}} & -\nu \\
-\nu & \frac{1}{1-D_{2}}
\end{array}\right]_{\left(\mathbf{d}_{1}, \mathbf{d}_{2}\right)}
$$

With this damage description, $\sqrt{2 E \Delta Y_{i}}=\Delta \Sigma_{i} /\left(1-D_{i}\right)$. The onset of coalescence is written as a damage localization condition. The number of cycles to coalescence $N_{\text {coal }}$ is obtained from the condition

$$
\mathrm{d}\left(\Delta \Sigma_{i}\right)=0 \quad \text { or equivalently } \quad \Delta \sigma_{i} \frac{\partial \ln \left(1-D_{i}\right)}{\partial \Delta \sigma_{i}}=-1
$$

that corresponds to the onset of strain and damage localization perpendicular to the considered eigen direction $\mathbf{d}_{i}[8]$.

\section{Example}

The mesoscopic initiation condition is described by using a damage model [9] written at a microscopic scale

$$
\frac{\delta D_{\mu}}{\delta N}=\left(\frac{\left\langle\Delta \sigma-\Delta \sigma_{\infty}\right\rangle}{\Delta S_{D}}\right)^{\eta}
$$

where $\Delta S_{D}$ and $\eta$ are material-dependent constants, and $D_{\mu}$ the microscopic damage variable. Since the microscopic yield stress $\sigma_{y}$ is equal to the local fatigue limit amplitude $\Delta \sigma_{\infty}$, the latter appears in Eqn. (11). Consequently, the number of cycles to initiation (i.e., when $D_{\mu}=1$ ) and the threshold stress become

$$
N_{I}\left(\Delta \sigma_{\infty}\right)=\left(\frac{\Delta S_{D}}{\left\langle\Delta \sigma-\Delta \sigma_{\infty}\right\rangle}\right)^{\eta} \text { and } \Delta \sigma_{u}(N)=\Delta S_{D} N^{-1 / \eta}
$$

When initiation occurs, the mesocrack size is equal to $a(0 ; \Delta \sigma)=\Phi\left(\Delta \sigma_{\infty}\right)$, where $\Phi$ is a characteristic size of the microstructure (e.g., grain size). If the Hall-Petch relationship applies, $\Delta \sigma_{\infty}\left(=\sigma_{y}\right)$ and $\Phi$ are related by

$$
\Phi\left(\Delta \sigma_{\infty}\right)=\left(\frac{K}{\Delta \sigma_{\infty}}\right)^{2}
$$

where $K$ is a material-dependent parameter. To get closed-form results, it is assumed that the mesocrack propagation is such that

$$
a\left(N-N_{I} ; \Delta \sigma\right)=\Phi\left(\Delta \sigma_{\infty}\right) \Psi\left(N-N_{I} ; \Delta \sigma\right),
$$

where the function $\Psi$ describes the propagation stage in a simple way. By definition, $\Psi(0 ; \Delta \sigma)=1$, and $\Psi(0 ; \Delta \sigma) \leq \Psi\left(N-N_{I} ; \Delta \sigma\right) \leq \Psi\left(N-N_{\min } ; \Delta \sigma\right)$, where the latter describes the propagation of the largest 
crack. Consequently, bounds to the obscuration probability and the density of mesocracks are derived. Bounds to the obscuration probability become $(m>2 n)$

$$
\begin{aligned}
& P_{\text {obs }}(N ; \Delta \sigma) \geq 1-\exp \left[-\frac{m}{m-2 n}\left\{\frac{\left\langle\Delta \sigma-\Delta \sigma_{u}(N)\right\rangle}{\Delta \sigma_{c}^{*}}\right\}^{m-2 n}\right] \\
& P_{\text {obs }}(N ; \Delta \sigma) \leq 1-\exp \left[-\frac{m}{m-2 n}\left\{\frac{\left\langle\Delta \sigma-\Delta \sigma_{u}(N)\right\rangle}{\Delta \sigma_{c}\left(N-N_{\min } ; \Delta \sigma\right)}\right\}^{m-2 n}\right],
\end{aligned}
$$

where $\Delta \sigma_{c}^{*}$ is a characteristic (initiation) stress depending upon the Weibull and Hall-Petch parameters and $\Delta \sigma_{c}$ is a characteristic (propagation) stress depending in addition on the crack propagation law

$$
\Delta \sigma_{c}^{*}=\Delta \sigma_{0}\left[\Delta \sigma_{0} \sqrt{a_{0}} / K\right]^{\frac{2 n}{m-2 n}} \quad \text { and } \quad \Delta \sigma_{c}\left(N-N_{\min } ; \Delta \sigma\right)=\Delta \sigma_{c}^{*}\left[\Psi\left(N-N_{\min } ; \Delta \sigma\right)\right]^{-\frac{n}{m-2 n}},
$$

with $a_{0}=\left(S \lambda_{0}\right)^{-1 / n}$ a reference crack size. Bounds to the density of mesocracks read $(m>2 n)$

$$
\begin{aligned}
& \frac{\lambda_{m}(N ; \Delta \sigma)}{\lambda_{c}\left(N-N_{\min } ; \Delta \sigma\right)} \geq\left(\frac{m-2 n}{m}\right)^{\frac{2 n}{m-2 n}} \gamma\left[\frac{m}{m-2 n} ; \frac{m}{m-2 n}\left\{\frac{\left\langle\Delta \sigma-\Delta \sigma_{u}(N)\right\rangle}{\Delta \sigma_{c}\left(N-N_{\min } ; \Delta \sigma\right)}\right\}^{m-2 n}\right] \\
& \frac{\lambda_{m}(N ; \Delta \sigma)}{\lambda_{c}^{*}} \leq\left(\frac{m-2 n}{m}\right)^{\frac{2 n}{m-2 n}} \gamma\left[\frac{m}{m-2 n} ; \frac{m}{m-2 n}\left\{\frac{\left\langle\Delta \sigma-\Delta \sigma_{u}(N)\right\rangle}{\Delta \sigma_{c}^{*}}\right\}^{m-2 n}\right],
\end{aligned}
$$

where $\gamma$ is the incomplete gamma function $\gamma[p, x]=\int_{0}^{x} t^{p-1} \exp (-t) \mathrm{d} t, \lambda_{c}^{*}$ and $\lambda_{c}$ characteristic densities

$$
\lambda_{c}^{*}=\lambda_{0}\left[\Delta \sigma_{0} \sqrt{a_{0}} / K\right]^{\frac{2 m n}{m-2 n}} \quad \text { and } \quad \lambda_{c}\left(N-N_{\min } ; \Delta \sigma\right)=\lambda_{c}^{*}\left[\Psi\left(N-N_{\min } ; \Delta \sigma\right)\right]^{-\frac{m n}{m-2 n}} .
$$

The characteristic quantities are related with one another by the following condition

$$
Z_{\text {obs }}(0 ; \Delta \sigma) \lambda_{t}\left[\Delta \sigma_{c}^{*}\right]=1 \text { and } Z_{\text {obs }}\left(N-N_{\min } ; \Delta \sigma\right) \lambda_{t}\left[\Delta \sigma_{c}\left(N-N_{\min } ; \Delta \sigma\right)\right]=1,
$$

i.e., the average number of site in a domain of measure $1 / \lambda_{c}^{*}\left(\right.$ resp., $\left.1 / \lambda_{c}\left(N-N_{\min } ; \Delta \sigma\right)\right)$ is equal to 1 .

An upper bound to the number of cycles to coalescence $N_{\text {coal }}$ is obtained from the condition

$$
D_{i}=1-\exp \left(-\frac{1}{m-2 n}\right) \quad \text { or }\left\langle\sqrt{2 E \Delta Y_{i}}-\Delta \sigma_{c}^{*} m^{-1 /(m-2 n)}\right\rangle=\Delta \sigma_{u}(N),
$$

leading to

$$
N_{\text {coal }}<\left[\frac{\Delta S_{D}}{\left\langle e^{1 /(m-2 n)} \Delta \Sigma-m^{-1 /(m-2 n)} \Delta \sigma_{c}^{*}\right\rangle}\right]^{\eta} .
$$

This type of analysis is valid as long as the horizon remains included in any examination zone. Otherwise, a weakest link hypothesis applies at the considered level.

\section{Perspectives}

The probabilistic model was described in the simple case of the initiation of cracks on a surface along two perpendicular directions. A more detailed analysis on the interaction between cracks aligned along any direction is needed. In thermomechanical fatigue, crack propagation is also driven by the stress profile induced by temperature variations through the thickness of a structure. This effect has also to be accounted for. 


\section{Acknowledgements}

This work was supported by the DOMZOME project. The authors wish to thank the anonymous reviewer for his/her valuable suggestions.

\section{References}

[1] J.M. Stephan, F. Curtit, C. Vindeirinho, S. Taheri, M. Akamatsu, C. Peniguel, Evaluation of the Risk of Damage in Mixing Zones: EDF R\&D Programme, Proceedings Fatigue 2002, (2002) 1707-1714.

[2] K.J. Miller, Materials Science Perspective of Metal Fatigue Resistance, Mat. Sci. Tech. 9 (1993) $453-462$.

[3] D. Jeulin, Modèles morphologiques de structures aléatoires et changement d'échelle, (thèse d'Etat, University of Caen, 1991).

[4] W. Weibull, A Statistical Theory of the Strength of Materials, Roy. Swed. Inst. Eng. Res. 151 (1939).

[5] M. Seyedi, F. Hild, Probabilistic Aspects in the Formation and Propagation of Multiple Crack Networks in Thermomechanical Fatigue, in: M.R. Bache, P.A. Blackmore, J. Draper, J.H. Edwards, P. Roberts, J.R. Yates, eds., Proceedings Fatigue 2003, EMAS, (2003) 495-502.

[6] C. Denoual, G. Barbier and F. Hild, A Probabilistic Approach for Fragmentation of Ceramics under Impact Loading, C. R. Acad. Sci. Paris 325 [Série IIb] (1997) 685-691.

[7] D. Jeulin and P. Jeulin, Synthesis of Rough Surfaces by Random Morphological Functions, Proceedings 3rd European Symposium of Stereology (1981) 239-246.

[8] F. Hild, P.-L. Larsson, F.A. Leckie, Localization due to Damage in two Direction Fiber-Reinforced Composites, ASME J. Appl. Mech. 63 (1996) 321-326.

[9] J. Lemaitre, A Course on Damage Mechanics, Springer-Verlag, Berlin (Germany), 1992. 
Corresponding author :

\section{François HILD}

LMT-Cachan

61, avenue du Président Wilson

94235 Cachan Cedex

Tel : 0147402254

Fax : 0147402240

Email : hild@lmt.ens-cachan.fr 